\title{
A Topological Representation Theorem for Tropical Oriented Matroids
}

\author{
Silke Horn (nèe Möser) \\ Discrete Optimization, TU Darmstadt, Germany
}

\begin{abstract}
Tropical oriented matroids were defined by Ardila and Develin in 2007. They are a tropical analogue of classical oriented matroids in the sense that they encode the properties of the types of points in an arrangement of tropical hyperplanes - in much the same way as the covectors of (classical) oriented matroids describe the types in arrangements of linear hyperplanes.

Not every oriented matroid can be realised by an arrangement of linear hyperplanes though. The famous Topological Representation Theorem by Folkman and Lawrence, however, states that every oriented matroid can be represented as an arrangement of pseudohyperplanes.

Ardila and Develin proved that tropical oriented matroids can be represented as mixed subdivisions of dilated simplices. In this paper I prove that this correspondence is a bijection. Moreover, I present a tropical analogue for the Topological Representation Theorem.

Résumé. La notion de matroïde orienté tropical a été introduite par Ardila et Develin en 2007. Ils sont un analogue des matroïdes orientés classiques dans le sens où ils codent les propriétés des types de points dans un arrangement d'hyperplans tropicaux - d'une manière très similaire à celle dont les covecteurs des matroïdes orientés (classiques) décrivent les types de points dans les arrangements d'hyperplans linéaires.

Tous les matroïdes orientés ne peuvent pas être représentés par un arrangement d'hyperplans linéaires. Cependant le célèbre théorème de représentation topologique de Folkman et Lawrence affirme que tout matroïde orienté peut être représenté par un arrangement de pseudo-hyperplans.

Ardila et Develin ont prouvé que les matroïdes orientés tropicaux peuvent être représentés par des sous-divisions mixtes de simplexes dilatés. Je prouve dans cet article que cette correspondance est une bijection. Je présente en outre, un analogue tropical du théorème de représentation topologique.
\end{abstract}

Keywords: combinatorics, oriented matroids, discrete topology, tropical geometry

\section{Introduction}

Tropical geometry is the study of algebraic geometry over the tropical semiring $(\mathbb{R}, \oplus, \otimes)$. A tropical hyperplane is the vanishing locus of a linear tropical polynomial.

From the combinatorial point of view though, a tropical hyperplane in $\mathbb{T}^{d-1}$ is just the (codimension1-skeleton of the) polar fan of the $(d-1)$-dimensional simplex $\triangle^{d-1}$. An arrangement of $n$ tropical hyperplanes in $\mathbb{T}^{d-1}$ induces a cell decomposition of $\mathbb{T}^{d-1}$ and each cell can be assigned a type that 
describes its position relative to each of the tropical hyperplanes. To be precise, the point $p$ is assigned the type $A=\left(A_{1}, \ldots, A_{n}\right)$ where $A_{i}$ denotes the set of closed sectors of the $i$-th tropical hyperplane in which $p$ is contained. See Figure 1(c) for an illustration.

Definition 1.1 (Cf. (Ardila and Develin, 2009, Definition 3.1)) For $n, d \geq 1$ an $(n, d)$-type is an $n$ tuple $\left(A_{1}, \ldots, A_{n}\right)$ of non-empty subsets of $[d]$.

An $(n, d)$-type $A$ can be represented as a subgraph $K_{A}$ of the complete bipartite graph $K_{n, d}$ : Denote the vertices of $K_{n, d}$ by $N_{1}, \ldots, N_{n}, D_{1}, \ldots, D_{d}$. Then the edges of $K_{A}$ are $\left\{\left\{N_{i}, D_{j}\right\} \mid j \in A_{i}\right\}$.

Besides tropical hyperplane arrangements there are other objects that share the notion of an $(n, d)$-type:

- If we label the vertices of $\triangle^{n-1}$ by $1, \ldots, n$, the vertices of the polytope $\triangle^{n-1} \times \triangle^{d-1}$ are in canonical bijection with the edges of the complete bipartite graph $K_{n, d}$. Then a cell $C$ in a subdivision of $\triangle^{n-1} \times \triangle^{d-1}$ is assigned the type corresponding to the subgraph of $K_{n, d}$ containing all edges that mark vertices of $C$. See e.g. De Loera et al. (2010) for a thorough treatment of triangulations.

- Given a mixed subdivision of $n \triangle^{d-1}$, every cell is a Minkowski sum of $n$ faces of $\triangle^{d-1}$. By identifying the faces of $\triangle^{d-1}$ with the subsets of $[d]$, this again yields an $(n, d)$-type. See Figure 1(a) for an example. We discuss mixed subdivisions in more detail in Section 3

- Tropical oriented matroids as defined in Ardila and Develin (2009) via a set of covector axioms generalise tropical hyperplane arrangements. We discuss them in Section 2

By Develin and Sturmfels (2004) regular subdivisions of $\triangle^{n-1} \times \triangle^{d-1}$ are dual to arrangements of $n$ tropical hyperplanes in $\mathbb{T}^{d-1}$. See Figure 1 for an illustration.

By the Cayley Trick ( $c f$. Huber et al. (2000) ) subdivisions of $\triangle^{n-1} \times \triangle^{d-1}$ are in bijection with mixed subdivisions of $n \triangle^{d-1}$.

By (Ardila and Develin, 2009. Thm. 6.3), the types of a tropical oriented matroid with parameters $(n, d)$ yield a subdivision of ${\triangle^{n-1}}^{n} \triangle^{d-1}$. They also conjecture this to be a bijection, i.e., that the types of the cells in any mixed subdivision of $n \triangle^{d-1}$ are the types of a tropical oriented matroid with parameters $(n, d)$. By (Ardila and Develin 2009, Prop. 6.4), these types satisfy the boundary, comparability and surrounding axioms. Thus, the only piece missing is the elimination axiom.

In Oh and Yoo (2010) it is proven that fine mixed subdivisions satisfy the elimination axiom.

In this paper we prove that the conjecture holds true in general:

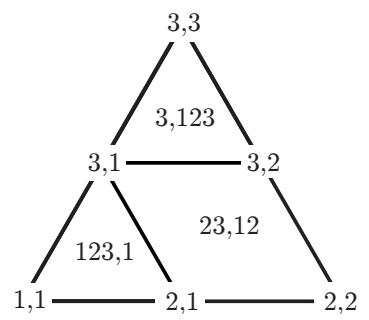

(a) A (regular) mixed subdivision of $2 \triangle^{2}$.

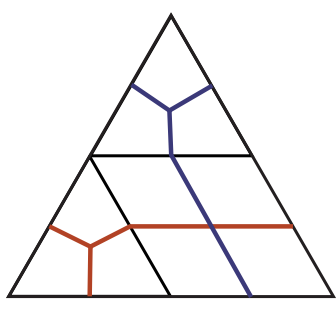

(b) The Poincaré dual of (a)

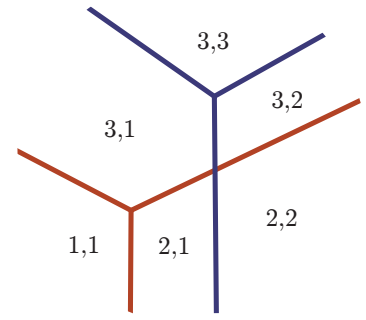

(c) An arrangement of tropical hyperplanes.

Fig. 1: The correspondence between mixed subdivisions and tropical pseudohyperplane arrangements. 
Theorem 1.2 (Cf. (Ardila and Develin, 2009, Conj. 5.1)) Tropical oriented matroids with parameters $(n, d)$ are in bijection with subdivisions of $\triangle^{n-1} \times \triangle^{d-1}$ and mixed subdivisions of $n \triangle^{d-1}$.

Moreover, we introduce arrangements of tropical pseudohyperplanes and prove a Topological Representation Theorem for tropical oriented matroids:

Theorem 1.3 (Topological Representation Theorem) Every tropical oriented matroid (in general position) can be realised by an arrangement of tropical pseudohyperplanes.

The paper is organised as follows: In Section 2 we briefly review the definition of tropical oriented matroids. Section 3 is dedicated to mixed subdivisions of dilated simplices. In Section 4 we introduce tropical pseudohyperplane arrangements and prove the Topological Representation Theorem. Finally, in Section 5 we apply our results to prove Theorem 1.2 .

\section{Tropical Oriented Matroids}

The following definitions are analogous to those in Ardila and Develin (2009).

A refinement of an $(n, d)$-type $A$ with respect to an ordered partition $P=\left(P_{1}, \ldots, P_{k}\right)$ of $[d]$ is the $(n, d)$-type $B=\left.A\right|_{P}$ where $B_{i}=A_{i} \cap P_{m(i)}$ and $m(i)$ is the smallest index where $A_{i} \cap P_{m(i)}$ is non-empty for each $i \in[n]$. A refinement is total if all $B_{i}$ are singletons.

Given $(n, d)$-types $A$ and $B$, the comparability graph $\mathrm{G}_{A, B}$ is a multigraph with node set $[d]$. For $1 \leq i \leq n$ there is an edge for every $j \in A_{i}, k \in B_{i}$. This edge is undirected if $j, k \in A_{i} \cap B_{i}$ and directed $j \rightarrow k$ otherwise. (We consider the comparability graph as a graph without loops.) Note that there may be several edges (with different directions) between two nodes.

A directed path in the comparability graph is a sequence $e_{1}, e_{2}, \ldots, e_{k}$ of incident edges at least one of which is directed and all directed edges of which are directed in the "right" direction. A directed cycle is a directed path whose starting and ending point agree. The graph is acyclic if it contains no directed cycle.

Definition 2.1 (Cf. Ardila and Develin, 2009, Definition 3.5)) $A$ tropical oriented matroid $M$ (with parameters $(n, d))$ is a collection of $(n, d)$-types which satisfies the following four axioms:

- Boundary: For each $j \in[d]$, the type $(j, j, \ldots, j)$ is in $M$.

- Comparability: The comparability graph $C_{G_{A, B}}$ of any two types $A, B \in M$ is acyclic.

- Elimination: If we fix two types $A, B \in M$ and a position $j \in[n]$, then there exists a type $C$ in $M$ with $C_{j}=A_{j} \cup B_{j}$ and $C_{k} \in\left\{A_{k}, B_{k}, A_{k} \cup B_{k}\right\}$ for $k \in[n]$.

- Surrounding: If $A$ is a type in $M$, then any refinement of $A$ is also in $M$.

We call $d=: \operatorname{rank} M$ the rank and $n$ the size of $M$.

Example 2.2 By (Ardila and Develin. 2009. Theorem 3.6) the set of types of an arrangement of $n$ tropical hyperplanes in $\mathbb{T}^{d-1}$ is a tropical oriented matroid with parameters $(n, d)$.

We call tropical oriented matroids coming from an arrangement of tropical hyperplanes realisable.

The axiom system was built to capture the features of the set of types in tropical hyperplane arrangements and thus the axioms have geometric interpretations: 
The boundary axiom ensures that all tropical hyperplanes in the arrangement are embedded correctly into $\mathbb{T P}^{d-1} \cong \triangle^{d-1}$. The surrounding axiom describes what the neighbourhood of a point of type $A$ (or equivalently, the star of the cell $A$ in the cell complex) looks like. The elimination axiom describes the intersection of a tropical line segment from $A$ to $B$ with the $j$-th tropical hyperplane. Finally, the comparability axiom ensures that we can declare a "direction from $A$ to $B$ ". Each position puts certain constraints on the direction vector, which may not contradict one another.

Definition 2.3 The dimension of an $(n, d)$-type $A$ is the number of connected components of $K_{A}$ minus 1. A vertex is a type of dimension 0 , an edge a type of dimension 1 and a tope a type of full dimension $d-1$, i.e., each tope is an n-tuple of singletons.

A tropical oriented matroid $M$ is in general position if for every type $A \in M$ the graph $K_{A}$ is acyclic.

For two types $A, B$ we write $A \supseteq B$ if $A_{i} \supseteq B_{i}$ for each $i \in[n]$. Moreover, we define the intersection $A \cap B:=\left(A_{1} \cap B_{1}, \ldots, A_{n} \cap B_{n}\right)$ and union $A \cup B:=\left(A_{1} \cup B_{1}, \ldots, A_{n} \cup B_{n}\right)$ of types.

Definition 2.4 (Cf. (Ardila and Develin, 2009, Propositions 4.7 and 4.8)) Let $M$ be a tropical oriented matroid with parameters $(n, d)$.

1. For $i \in[n]$ the deletion $M_{\backslash i}$ consisting of all $(n-1, d)$-types which arise from types of $M$ by deleting coordinate $i$ is a tropical oriented matroid with parameters $(n-1, d)$.

2. For $j \in[d]$ the contraction $M_{/ j}$ consisting of all types of $M$ that do not contain $j$ in any coordinate is a tropical oriented matroid with parameters $(n, d-1)$.

\section{Mixed Subdivisions}

Given two sets $X, Y$ their Minkowski sum $X+Y$ is given by $X+Y:=\{x+y \mid x \in X, y \in Y\}$.

Definition 3.1 Let $P_{1}, \ldots, P_{k} \subset \mathbb{R}^{n}$ be (full-dimensional) convex polytopes. Then a polytopal subdivision $\left\{Q_{1}, \ldots, Q_{s}\right\}$ of $P:=\sum P_{i}$ is a mixed subdivision if it satisfies the following conditions:

1. Each $Q_{i}$ is a Minkowski sum $Q_{i}=\sum_{j=1}^{k} F_{i, j}$, where $F_{i, j}$ is a face of $P_{j}$.

2. For $i, j \in[s]$ we have that $Q_{i} \cap Q_{j}=\left(F_{i, 1} \cap F_{j, 1}\right)+\ldots+\left(F_{i, k} \cap F_{j, k}\right)$.

Note that this definition can easily be generalised for polytopes which are not full-dimensional.

Let $S, S^{\prime}$ be mixed subdivisions of $n \triangle^{d-1}$. Then we say that $S^{\prime}$ is a refinement of $S$ if for every cell $C^{\prime} \in S^{\prime}$ there is a cell $C \in S$ such that $C^{\prime} \subseteq C$. This defines a partial order on the set of mixed subdivisions of $n \triangle^{d-1}$. A mixed subdivision is fine if there is no mixed subdivision refining it.

\subsection{Mixed Subdivisions of $n \triangle^{d-1}$}

We are interested in the case of mixed subdivisions where $P_{i}=\triangle^{d-1}$ for each $i$. Then $\sum P_{i}=n \triangle^{d-1}$ is a dilated simplex. By (Ardila and Develin. 2009. Theorem 6.3) the types of a tropical oriented matroid with parameters $(n, d)$ yield a mixed subdivision of $n \triangle^{d-1}$. A tropical oriented matroid is in general position if and only if its mixed subdivision is fine.

If $Q=\sum_{i=1}^{n} F_{i}$, where $F_{i} \subset[d]$ is a cell in such a mixed subdivision then we call $\left(F_{1}, F_{2}, \ldots, F_{n}\right)$ its type and denote it by $\mathcal{T}_{Q}$. Note that this is an $(n, d)$-type as defined in Definition 1.1. Conversely, if we are given an $(n, d)$-type $A$ then this corresponds to a unique cell inside $n \triangle^{d-1}$, which we denote by $\mathcal{C}_{A}$. 
In general, we call a cell corresponding to an $(n, d)$-type, i.e., a Minkowski sum of $n$ faces of $\triangle^{d-1}$, a Minkowski cell.

To avoid confusion with the vertices of tropical oriented matroids, we speak of the 0-dimensional cells of a mixed subdivision as topes.

There is a canonical embedding of a mixed subdivision of $n \triangle^{d-1}$ into $\mathbb{R}^{d}$ (by mapping a tope $v$ to $\left(x_{1}, \ldots, x_{d}\right)$ where $x_{i}$ is the number of occurences of $i$ in $\left.v\right)$. We thus regard a mixed subdivision - or any subset of its (open) cells - as a metric space with the Euclidean metric inherited from $\mathbb{R}^{d}$.

We now establish some properties of mixed subdivisions of $n \triangle^{d-1}-$ or more generally about $(n, d)$ types. Note that since we can describe the Minkowski cells in a mixed subdivision of $n \triangle^{d-1}$ in terms of $(n, d)$-types, we can transfer properties of tropical oriented matroids (such as the boundary, surrounding, comparability or elimination property) as defined in Section 2 to mixed subdivisions of $n \triangle^{d-1}$.

Lemma 3.2 Let $A, B$ be two $(n, d)$-types with $A \subseteq B$. Then $A$ is a refinement of $B$ if and only if $C_{G}, B$ is acyclic.

Lemma 3.3 Let $A, B$ be two types in a mixed subdivision $S$ of $n \triangle^{d-1}$. Then their intersection $A \cap B$ either has an empty position or is also a type in $S$.

Lemma 3.4 Given a Minkowski cell $Q=\sum_{i=1}^{n} F_{i}$ in a mixed subdivision of $n \triangle^{d-1}$ then the faces of $Q$ are exactly the $\mathcal{C}_{R}$ where $R$ is a refinement of $\mathcal{T}_{Q}$.

Lemma 3.5 Let $A, B$ be $(n, d)$-types such that $C_{G_{A, B}}$ is acyclic. Then $\mathcal{C}_{A} \cap \mathcal{C}_{B}=\mathcal{C}_{A \cap B}$.

We can define the concepts of deletion and contraction for mixed subdivisions analogous to Definition 2.4 The following observations are immediate:

Lemma 3.6 Let $S$ be a mixed subdivision of $n \triangle^{d-1}$.

1. For any $i \in[n]$ the deletion $S_{\backslash i}$ is a mixed subdivision of $(n-1) \triangle^{d-1}$.

2. For any $j \in[d]$ the contraction $S_{/ j}$ is a mixed subdivision of $n \triangle^{d-2}$.

\subsection{Reconstructing Mixed Subdivisions}

In this section we prove the following:

Proposition 3.7 Let $S$ be a mixed subdivision of $n \triangle^{d-1}$. Then $S$ can be reconstructed from its topes.

More precisely, the cells of $S$ are exactly the unions of topes all of whose total refinements are topes and which do not contain any other tope.

Note that it is crucial to consider the topes of $S$ as types rather than as mere coordinates; i.e., the order of the summands matters here. Also note that the equivalent result for tropical oriented matroids, namely that a tropical oriented matroid is uniquely determined by its topes, is proven in Ardila and Develin (2009). Their proof, however, uses the elimination property.

Proof: We call the types satisfying the conditions above the nice types of $S$. It is clear that all cells are nice. So it remains to prove that every nice type does actually yield a cell of $S$.

The general strategy is the following: Assume that $A$ is a nice cell in $S$. We argue that $A$ intersects every cell $B$ of $S$ either not at all or in a common face of $A$ and $B$, proving that $A$ is in fact a cell in $S$. By Lemma 3.5 it suffices to prove that (the types of) $A$ and $B$ are comparable. 
For $i \in[n]$ consider the deletion map

$$
\cdot{ }_{\backslash i}: S \rightarrow S_{\backslash i}: C \mapsto C_{\backslash i}=\left(C_{1}, \ldots, \widehat{C_{i}}, \ldots, C_{n}\right)
$$

mapping each cell $C$ of $S$ to the cell obtained by omitting the $i$-th entry of $C$.

Lemma 3.8 Let $S$ be a mixed subdivision of $n \triangle^{d-1}, i \in[n]$ and $A \neq B$ types of cells $\mathcal{C}_{A}, \mathcal{C}_{B} \in S$ cells such that $A_{\backslash i}=B_{\backslash i}$. Then $A \cup B$ is the type of a cell in $S$.

Proof: Let $C:=A \cup B$, i.e., $C_{i}:=A_{i} \cup B_{i}$ and $C_{j}=A_{j}\left(=B_{j}\right)$ for each $j \neq i$. The situation is sketched in Figure 2. The intuition is that $\mathcal{C}_{C}$ (unless $C$ already equals $A$ or $B$ ) is a prism over $\mathcal{C}_{A}\left(\right.$ or $\left.\mathcal{C}_{B}\right)$ with $\mathcal{C}_{A}$ and $\mathcal{C}_{B}$ the top, respectively bottom face of $\mathcal{C}_{C}$.

We need to show that $\mathcal{C}_{C}$ is indeed a cell in $S$. To this end, we verify that $C$ satisfies the conditions from Proposition 3.7 This means we have to show that the total refinements of $C$ are exactly the total refinements of $A$ and $B$.

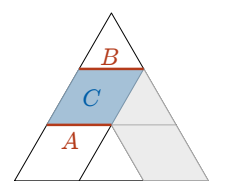

Fig. 2: The two edges $A$ and $B$ are mapped to the same cell under the deletion map that deletes the shaded cells.

\subsection{Convexity in tropical oriented matroids}

One can exploit the elimination property of tropical oriented matroids to obtain topological properties of the according mixed subdivisions.

Let $M$ be a tropical oriented matroid and $A, B \in M$ two types. Then the set

$$
M_{A B}:=\left\{C \in M \mid C_{i} \in\left\{A_{i}, B_{i}, A_{i} \cup B_{i}\right\} \text { for all } i \in[n]\right\}
$$

is the (combinatorial) convex hull of $A$ and $B$. Analogously we define the (combinatorial) convex hull $S_{A B}$ of two cells in a mixed subdivision $S$ of $n \triangle^{d-1}$.

We say that a subset $C$ of a tropical oriented matroid $M$ (or equivalently, a subcomplex of a mixed subdivision of $n \triangle^{d-1}$ ) is convex if for any $A, B \in C$ we have that $M_{A B} \subseteq C$.

Proposition 3.9 The types of the cells in a mixed subdivision $S$ of $n \triangle^{d-1}$ satisfy the elimination property if and only if $S_{A B}$ is path-connected (as a subcomplex of $S$ ) for every $A, B \in S$.

Proof: If $S_{A B}$ is path-connected then there is a path from $A$ to $B$ in $S_{A B}$. For any given $j \in[n]$ this path must contain a cell $C$ with $C_{j}=A_{j} \cup B_{j}$. Then $C$ works as elimination for $A$ and $B$ with respect to $j$. The converse can be shown by induction on the cardinality of $\operatorname{dist}(A, B):=\left\{i \mid A_{i} \not \subseteq B_{i}, B_{i} \nsubseteq \subseteq A_{i}\right\}$.

Corollary 3.10 A convex set in a tropical oriented matroid is path-connected. 


\section{The Topological Representation Theorem}

This section comprises the long and winding road towards the Topological Representation Theorem for tropical oriented matroids.

\subsection{Tropical Pseudohyperplanes}

Definition 4.1 A tropical pseudohyperplane is the image of a tropical hyperplane under a PL-homeomorphism of $\mathbb{P P}^{d-1}$ that fixes the boundary.

The following theorem is a crucial ingredient to the proof of the Topological Representation Theorem: In an arrangement of tropical hyperplanes, the $i$-th tropical hyperplane consists exactly of those points $A$ with $\# A_{i} \geq 2$. We show the analogue for the Poincaré dual of a mixed subdivision of $n \triangle^{d-1}$.

Theorem 4.2 Let $S$ be a mixed subdivision of $n \triangle^{d-1}$ and $i \in[n]$. Then $\left\{C^{*} \mid C \in S, \# C_{i} \geq 2\right\}$ is a tropical pseudohyperplane.

Proof: We prove the claim by induction over $n$. For $n=1$ this is true since then $S=\triangle^{d-1}$ is the trivial subdivision, whose dual is the cell complex of one $(d-2)$-dimensional tropical hyperplane in $\mathbb{T}^{d-1}$.

Now assume $n \geq 2$. Choose $i \neq j \in[n]$ and consider the deletion $S_{\backslash j}$. By Lemma 3.6 this is a mixed subdivision of $(n-1) \triangle^{d-1}$ and by induction the image of $H_{i}$ in $S_{\backslash j}$ is a tropical pseudohyperplane $h$.

But $H_{i}$ is the preimage of $h$ under the deletion map. By Lemma 3.8 this preimage is PL-homeomorphic to $h$ and hence a tropical pseudohyperplane.

\subsection{Linear and affine pseudohyperplanes}

Locally, (i.e., in the parallelepiped cells of their mixed subdivisions) we want tropical pseudohyperplanes to intersect as "ordinary" hyperplanes. We thus introduce arrangements of linear pseudohyperplanes on the basis of arrangements of pseudospheres as defined in (Björner et al., 1999, Def. 5.1.3).

Definition 4.3 A finite collection $\mathcal{A}=\left(H_{e}\right)_{e \in E}$ of pseudohyperplanes is called an arrangement of pseudohyperplanes if the following conditions hold:

1. $H_{A}:=\bigcap_{e \in A} H_{e}$ is a pseudohyperplane of smaller dimension for all $A \subseteq E$.

2. If $H_{A} \nsubseteq H_{e}$ for $A \subseteq E, e \in E$ and $H_{e}^{+}$and $H_{e}^{-}$are the two sides of $H_{e}$, then $H_{A} \cap H_{e}$ is a pseudohyperplane in $H_{A}$ with sides $H_{A} \cap H_{e}^{+}$and $H_{A} \cap H_{e}^{-}$.

3. The intersection of an arbitrary collection of closed sides is a ball.

We now define arrangements of affine pseudohyperplanes as a generalisation of the above:

Definition 4.4 An arrangement of affine pseudohyperplanes is a collection $\mathcal{A}$ of (linear) pseudohyperplanes such that for any $\mathcal{A}^{\prime} \subseteq \mathcal{A}$ either $\bigcap_{a \in \mathcal{A}^{\prime}} H_{a}=\emptyset$ or $\mathcal{A}^{\prime}$ is an arrangement of linear pseudohyperplanes as defined in Definition 4.3

Lemma 4.5 The intersection of any number of closed affine pseudohalfspaces in $\mathbb{R}^{d}$ is path-connected. 


\subsection{Arrangements of tropical pseudohyperplanes}

Let $H$ be a $(d-2)$-dimensional tropical pseudohyperplane in $\mathbb{T}^{d-1}$. Then $H$ divides $\mathbb{T}^{d-1} \backslash H$ into $d$ connected components $S_{1}, \ldots, S_{d}$, the open sectors of $H$. The closure of any union $\bigcup_{i \in I} S_{i}$ with $\emptyset \neq I \subset[d]$ will be called a pseudohalfspace of $H$. We denote by

$$
H_{I}:=\partial \overline{\bigcup_{i \in I} S_{i}}=\partial \overline{\bigcup_{i \notin I} S_{i}}
$$

the boundary of the pseudohalfspace and by

$$
H_{I}^{+}:=\overline{\bigcup_{i \in I} S_{i}} \backslash H_{I}, \quad \text { respectively } \quad H_{I}^{-}:=\overline{\bigcup_{i \notin I} S_{i}} \backslash H_{I}
$$

the two open pseudohalfspaces.

An $(n, d)$-halfspace system is a tuple $\mathcal{I}=\left(I_{1}, \ldots, I_{n}\right)$ with $\emptyset \neq I_{i} \subset[d]$ for each $1 \leq i \leq n$. Given a halfspace system $\mathcal{I}$ and a collection $\mathcal{A}=\left(H_{i}\right)_{i \in[n]}$ of $n$ tropical pseudohyperplanes we write

$$
\mathcal{A}_{\mathcal{I}}:=\left\{H_{i, I_{i}} \mid 1 \leq i \leq n\right\} .
$$

We can now state the definition of tropical pseudohyperplane arrangements:

Definition 4.6 An arrangement of tropical pseudohyperplanes (in weakly general position) is a collection $\mathcal{A}$ of $n$ tropical pseudohyperplanes in $\mathbb{T}^{d-1}$ such that $\mathcal{A}_{\mathcal{I}}$ forms an arrangement of affine pseudohyperplanes as defined in Definition 4.4 for every $(n, d)$-halfspace system $\mathcal{I}$.

For a halfspace $I$, i.e., $\emptyset \neq I \subset[d]$, of a tropical pseudohyperplane $H$ we define the map $\mathcal{T}_{I}$ that maps a cell $C$ of $H$ to + if $C \subseteq I$, to - if $C \subseteq \bar{I}$ and to 0 if $C \cap I, C \cap \bar{I} \neq \emptyset$. Now let $\mathcal{A}$ be a tropical pseudohyperplane arrangement and $\mathcal{C}(\mathcal{A})$ the induced cell decomposition of $\mathbb{T}^{d-1}$. For $\mathcal{A}^{\prime} \subseteq \mathcal{A}$ we define

$$
\begin{aligned}
\mathcal{T}_{\mathcal{I}}: \mathcal{C}\left(\mathcal{A}^{\prime}\right) & \rightarrow\{+,-, 0\} \mathcal{A}^{\prime}: \\
C & \mapsto\left(\mathcal{T}_{I_{i}}\left(C_{i}\right)\right)_{i} .
\end{aligned}
$$

Now let $\mathcal{J}=\left(J_{1}, \ldots, J_{n}\right)$ be an $n$-tuple of partitions of $[d]$. I.e., each $J_{i}=\left(J_{i, 1} \cup \ldots \cup J_{i, k_{i}}\right)$ is a partition of $[d]$ for each $i \in[n]$. For a tropical oriented matroid $M$ denote by

$$
M_{\mathcal{J}}:=\left\{A \in M \mid A_{i} \cap J_{i, k} \neq \emptyset, i \in[n], k \in\left[k_{i}\right]\right\}
$$

the set containing all types in $M$ all of whose entries intersect each element in the according partition. As before, let $\mathcal{I}=\left(I_{1}, \ldots, I_{n}\right)$ be an $n$-tuple of non-empty subsets of $[d]$. Then we denote

$$
M_{\mathcal{I}}:=\left\{A \in M \mid A_{i} \subseteq I_{i}, i \in[n]\right\} .
$$

Finally, we define

$$
M(\mathcal{I}, \mathcal{J}):=M_{\mathcal{I}} \cap M_{\mathcal{J}} .
$$

The following is a consequence of Corollary 3.10 .

Lemma 4.7 Let $M$ be a tropical oriented matroid in general position. Then $M(\mathcal{I}, \mathcal{J})$, if non-empty, is connected and pure of dimension $d+n-1-\sum \# J_{i}$. 
For a cell complex $\mathcal{C}$ we denote by $\overline{\mathcal{C}}$ its closure, i.e., $\overline{\mathcal{C}}$ consists of all cells of $\mathcal{C}$ and their faces.

Lemma 4.8 Let $M, \mathcal{I}, \mathcal{J}$ as before. Then $\overline{M(\mathcal{I}, \mathcal{J})}$ is a PL-manifold with boundary.

Proof: Denote $\mathcal{M}:=\overline{M(\mathcal{I}, \mathcal{J})}$ and $\mathcal{M}^{\prime}:=M_{\mathcal{J}}$. Choose a cell $T \in \mathcal{M}$. We first investigate the link $\operatorname{lk}_{\mathcal{M}^{\prime}} T$. The cells in $\mathrm{lk}_{\mathcal{M}^{\prime}} T$ correspond to the cells in the star st $\mathcal{M}^{\prime} T=\left\{C \in \mathcal{M}^{\prime} \mid C \subseteq T\right\}$ and hence to certain refinements of $T$. First assume that $n=1=k_{1}$, i.e., $\mathcal{J}=\left(J_{1}=\left(J_{11}\right)\right)$. Then the cells in st $_{\mathcal{M}^{\prime}} T$ are in bijection with the proper subsets of $J_{11} \cap T_{1}$ ordered by reverse inclusion. Hence $\mathrm{lk}_{\mathcal{M}^{\prime}} T$ is the boundary of a simplex of dimension \# $\left(J_{11} \cap T_{1}\right)-1$. Since $M$ is in general position we can consider the $J_{i k}$ (for $i \in[n], k \in\left[k_{i}\right]$ ) independently. I.e., in general, $\operatorname{lk}_{\mathcal{M}} T$ is the boundary of a product of simplices (one for each $J_{i k}$ ). Denote this sphere by $\mathcal{S}(T)$. See Figures 3(b) and (c) for an example.

If in each position $i$ there is some $J_{i k}$ with $J_{i k} \cap T_{i} \subseteq I_{i}$ then $T$ is contained in the interior of $\mathcal{M}$ and $\operatorname{lk}_{\mathcal{M}} T=\mathcal{S}(T)$. Otherwise denote by $\mathcal{B}(T)$ the set of all faces of $\mathcal{S}(T)$ that do not belong to $\mathrm{lk}_{\mathcal{M}} T$. Then define $\mathcal{J}^{\prime}$ by replacing each $J_{i}$ in $\mathcal{J}$ by $\left(I_{i} \bullet\left(J_{i 1} \cap \overline{I_{i}}\right) \cup \ldots \cup\left(J_{i k_{i}} \cap \overline{I_{i}}\right)\right)$. Then $\overline{\mathcal{B}(T)} \cap \operatorname{lk}_{\mathcal{M}} T=M_{\mathcal{J}^{\prime}}$ is a PL-sphere in $\mathcal{S}(T)$ with sides $\mathcal{B}(T)$ and $\operatorname{lk}_{\mathcal{M}} T$. This implies that $\operatorname{lk}_{\mathcal{M}} T$ is a PL-ball.

Moreover, $\mathcal{M}$ has a boundary since - unless $\mathcal{M}$ consists of a single point or contains a cell whose link is a ball - we can always construct a cell in $\mathcal{M}$ whose dual is contained in the boundary of $n \triangle^{d-1}$.

\subsection{Constructibility}

The notion of constructibility of a polytopal complex goes back to Hochster (1972).

Definition 4.9 A polyhedral d-complex $C$ is constructible if $C$ consists of only one cell or $C=C_{1} \cup$ $C_{2}$, where $C_{1}, C_{2}$ are d-dimensional constructible complexes and $C_{1} \cap C_{2}$ is a $(d-1)$-dimensional constructible complex.

Proposition 4.10 Let $M, \mathcal{I}, \mathcal{J}$ as before. Then $M(\mathcal{I}, \mathcal{J})$ is constructible.

Proof: We are done if $M(\mathcal{I}, \mathcal{J})$ consists of one (maximal) cell only. Otherwise there are two maximal cells $A$ and $B$. By Lemma 4.7 above (and the fact that $A, B$ are maximal) we then have $\# A_{i}=\# B_{i}$ and $\# A_{i} \cap J_{i, j}=\# B_{i} \cap J_{i, j}=1$ for every $i$ and $j$.

There is some position $k$ where $A$ and $B$ differ. Moreover, there is some $\ell$ with $J_{k, \ell} \cap A_{k} \neq J_{k, \ell} \cap B_{k}$. Let $a \in J_{k, \ell} \cap A_{k}, b \in J_{k, \ell} \cap B_{k}$. (Note that $a$ and $b$ are unique.)

Now form $\mathcal{J}_{0}$ by splitting $J_{k, \ell}$ so that $a$ and $b$ are in different sets. Moreover, form $\mathcal{I}_{1}, \mathcal{I}_{2}$ by removing $a$, respectively $b$ from $I_{k}$. Then $\overline{M(\mathcal{I}, \mathcal{J})}=\overline{M\left(\mathcal{I}_{1}, \mathcal{J}\right)} \cup \overline{M\left(\mathcal{I}_{2}, \mathcal{J}\right)}$ and $\overline{M\left(\mathcal{I}_{1}, \mathcal{J}\right)} \cap \overline{M\left(\mathcal{I}_{2}, \mathcal{J}\right)}=\overline{M\left(\mathcal{I}, \mathcal{J}_{0}\right)}$. Moreover, $A \in \overline{M\left(\mathcal{I}_{1}, \mathcal{J}\right)}, B \in \overline{M\left(\mathcal{I}_{2}, \mathcal{J}\right)}$. By the above lemma, $M\left(\mathcal{I}_{1}, \mathcal{J}\right), M\left(\mathcal{I}_{2}, \mathcal{J}\right), M\left(\mathcal{I}, \mathcal{J}_{0}\right)$ are connected and pure and of the right dimensions. By induction these three sets are constructible and hence $\overline{M(\mathcal{I}, \mathcal{J})}$ is constructible. See Figure 3 for an illustration.

The above lemmas together with a theorem by Zeeman (Zeeman (1963), "A constructible manifold with a boundary is a ball.") yield:

Proposition 4.11 Let $M$ be a tropical oriented matroid in general position. Then $M(\mathcal{I}, \mathcal{J})$ is a PL-ball.

We are now ready to prove the following version of the Topological Representation Theorem for tropical oriented matroids:

Theorem 4.12 Every tropical oriented matroid in general position can be realised by an arrangement of tropical pseudohyperplanes as in Definition 4.6. 


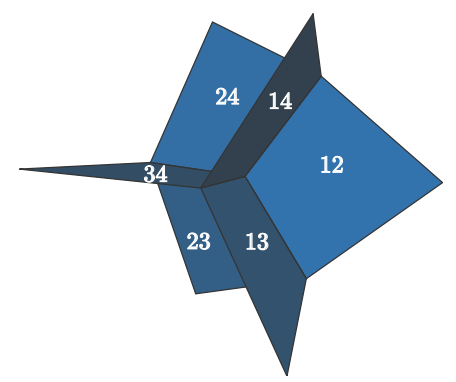

(a) A 2-dimensional tropical pseudohyperplane. The 2 -faces are labelled by their types.

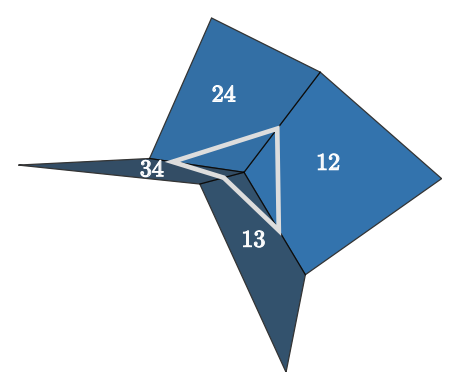

(b) The subcomplex $M(\mathcal{I}, \mathcal{J})$ for $\mathcal{I}=[4], \mathcal{J}=(14 \bullet 23)-$ a 2 dimensional PL-ball. The link of 1234 is drawn in light grey.

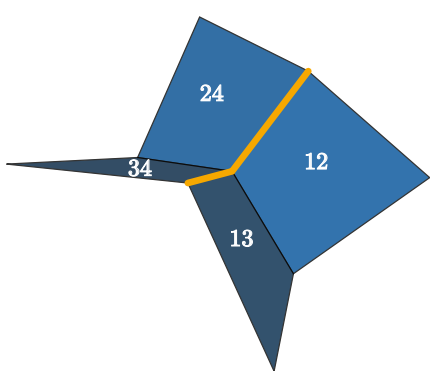

(c) The subcomplex $M\left(\mathcal{I}, \mathcal{J}_{0}\right)$ for $\mathcal{I}=[4], \mathcal{J}_{0}=(1 \cup 4 \cup 23)-\mathrm{a}$ 1-dimensional PL-ball - and its sides $M\left(\mathcal{I}_{1}, \mathcal{J}\right)$ and $M\left(\mathcal{I}_{2}, \mathcal{J}\right)$.

Fig. 3: Assume in the proof of Proposition 4.10 we have $n=1, d=4$, i.e., we are dealing with a 2-dimensional tropical pseudohyperplane as depicted in Figure (a) Moreover, assume we have $M(\mathcal{I}, \mathcal{J})$ with $\mathcal{I}=[4], \mathcal{J}=$ $(14 \bullet 23)$. The complex $M(\mathcal{I}, \mathcal{J})$ is depicted in Figure (b)

Now let $A=13, B=24$. As in the proof we see that $\# A_{1}=\# B_{1}$ and $\# A_{1} \cap J_{1 i}=\# B_{1} \cap J_{1 j}=1$ for every $i$ and $j$. We have $k=1$ and we may choose $\ell=1$. Then we get $a=1, b=4$ as the unique elements in $A_{1} \cap J_{11}, B_{1} \cap J_{11}$. We form $\mathcal{J}_{0}=(1 \cup 4 \cup 23)$ by splitting $J_{k \ell}=14$. Moreover, we set $\mathcal{I}_{1}=234$ and $\mathcal{I}_{2}=123$. This situation is depicted in Figure (c)

Proof: Let $M$ be a tropical oriented matroid in general position, $S$ the fine mixed subdivision of $n \triangle^{d-1}$ corresponding to $M$ and $\mathcal{A}$ the family of tropical pseudohyperplanes induced by $S$. We have to show that $\mathcal{A}_{\mathcal{I}}^{\prime}$ is an arrangement of affine pseudohyperplanes for each $\mathcal{A}^{\prime} \subseteq \mathcal{A}$ and halfspace system $\mathcal{I}$.

So assume that $\bigcap \mathcal{A}_{\mathcal{I}}^{\prime} \neq \emptyset$. We have to show that $\mathcal{A}_{\mathcal{I}}^{\prime}$ satisfies the axioms in Definition 4.3 Each axiom comes down to verifying that a certain set is a PL-ball. Each of these sets can be represented as $M(\mathcal{I}, \mathcal{J})$ for suitable $\mathcal{I}$ and $\mathcal{J}$.

\section{The elimination property}

This section is about the all important elimination property. Recall that by (Oh and Yoo, 2010, Prop. 4.12) the elimination property holds for fine mixed subdivisions of $n \triangle^{d-1}$. In this section we apply the Topological Representation Theorem 4.12 to extend this to all mixed subdivisions of $n \triangle^{d-1}$.

\subsection{Blowing up hyperplanes in a mixed subdivision}

Let $S$ be a fine mixed subdivision of $n \triangle^{d-1}$ and fix $i \in[n]$. The following construction is an inverse of the deletion operation and yields a mixed subdivision of $N^{d-1}(N>n)$ by "blowing up" one tropical pseudohyperplane in the dual arrangement. The construction can be defined for any mixed subdivision of $n \triangle^{d-1}$. For the sake of brevity, however, we only present the construction for fine ones.

Let $S, S^{\prime}$ be fine mixed subdivisions of $n \triangle^{d-1}$, respectively $n^{\prime} \triangle^{d-1}$. Then the blow-up up $S$ with respect to $S^{\prime}$ at position $i$ is

$$
S \vee_{i} S^{\prime}:=\bigcup_{C \in S}\left\{\left(C_{\backslash i}, X\right) \mid X \in S_{\backslash \bar{C}_{i}}^{\prime}\right\}
$$


I.e., we subdivide the $i$-th summand of each cell $C \in S$ as the $C_{i}$-face of $S^{\prime}$. See Figure 4 for an example. The following lemma follows easily:

Lemma 5.1 The types in the blow-up $S \vee_{i} S^{\prime}$ yield a fine mixed subdivision of $\left(n+n^{\prime}-1\right) \triangle^{d-1}$.

\subsection{Elimination in mixed subdivisions}

In this section we prove that tropical pseudohyperplane arrangements as defined in Definition 4.6 satisfy the elimination property and use this to show the same for all mixed subdivisions of $n \triangle^{d-1}$.

Since it simplifies the presentation we assume all arrangements of tropical pseudohyperplanes in this section to come from a (fine) mixed subdivision of $n \triangle^{d-1}$. I.e., we only consider tropical pseudohyperplane arrangements which are dual to a fine mixed subdivision of $n \triangle^{d-1}$.

Let $H$ be a tropical hyperplane with apex 0 . Recall that $H_{I}$ denotes the boundary of the tropical halfspace separating the points with types in $I$ from those with types in the complement $\bar{I}$. For $p \in \mathbb{T}^{d-1}$ and $\emptyset \neq I \subseteq[d]$ denote $H_{I, p}:=H_{I}-p$, i.e., we shift the apex of $H_{I}$ to $p$. For $\emptyset \neq I \subseteq[d]$ denote by $T_{I}$ the set of all points of type $I$. For $X \subseteq\{I \mid \emptyset \neq I \subset[d]\}$ we say that $A \subseteq \mathbb{T}^{d-1}$ approximates $T_{X}:=\bigcup_{I \in X} T_{I}$ if:

- For each $I \in X$, there is $\varepsilon_{I}>0$ such that $T_{I}$ is contained in $A$ except possibly for an $\varepsilon_{I^{-}}$ neighbourhood of the (relative) boundary $\partial T_{I}$.

- For each $I \notin X$ there is $\varepsilon_{I}>0$ such that $T_{I} \cap A$ is contained in an $\varepsilon_{I}$-neighbourhood of $\partial T_{I}$.

Intuitively, the set $A$ is supposed to contain "almost everything" of $T_{I}$ if $I \in X$ and "almost nothing" of $T_{I}$ if $I \notin X$. Then $T_{X}$ is homeomorphic to deformation retract of $A$. We will be interested in approximating neighbourhoods for $X=\{a, b, a \cup b\}$ with $a, b \subset[d]$. See Figure 5 for an illustration.

The proof of the following is rather technical and omitted for the sake of brevity.

Lemma 5.2 Let $H$ be a tropical hyperplane in $\mathbb{T}^{d-1}$ and $\emptyset \neq I, I^{\prime} \subset[d]$. Then we can construct an approximating neighbourhood of $T_{I} \cup T_{I^{\prime}} \cup T_{I \cup I^{\prime}}$ as an intersection of shifted pseudohalfspaces $H_{J, p}^{+}$. Moreover, these pseudohalfspaces can be constructed explicitly by suitable blow-ups of $H$.

See Figure 6 for an example.

We can extend the above construction to arrangements of tropical pseudohyperplanes.

Lemma 5.3 Let $\mathcal{A}=\left(H_{i}\right)_{i \in[n]}$ be an arrangement of tropical pseudohyperplanes dual to a mixed subdivision $S$ of $n \triangle^{d-1}$. Fix two halfspace systems $\mathcal{I}, \mathcal{I}^{\prime}$. Then we can construct an approximating neighbourhood $A$ of $E:=\bigcap\left(H_{i, I_{i}} \cap H_{i, I_{i}^{\prime}}\right)$ by repeated blow-ups (for each position) of $S$. Moreover, $A$ is homeomorphic to a deformation retract of $E$.
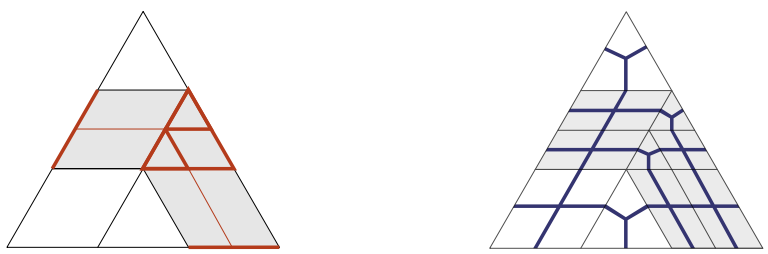

Fig. 4: The blow-up of a mixed subdivision of $3 \triangle^{2}$ with respect to one of $2 \triangle^{2}$. The cells in the shaded hyperplane are subdivided according to the subdivision of the small simplex. The according tropical pseudohyperplane arrangement is drawn on the left. 

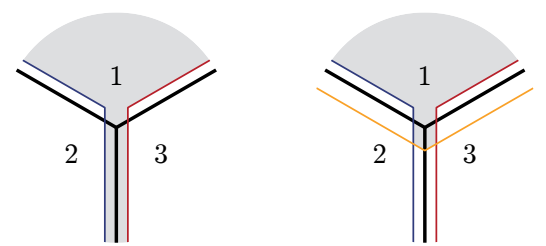

Fig. 5: Approximating neighbourhoods corresponding to $a=1$ and $b=23$ (on the left), respectively $a=1$ and $b=123$ (on the right).

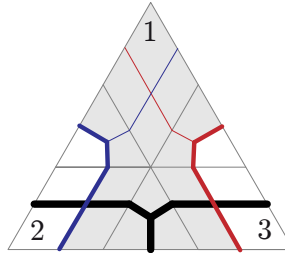

Fig. 6: An approximating neighbourhood for $a=1, b=$ 23 as an intersection of affine pseudohalfspaces in a blowup of the black tropical pseudohyperplane.

Theorem 5.4 Every mixed subdivision of $n \triangle^{d-1}$ satisfies the elimination property.

Proof: Let $S$ be a mixed subdivision of $n \triangle^{d-1}$. By Proposition 3.9 it suffices to show that $S_{A B}$ is connected for any two cells $A, B \in S$. By Lemmas 5.2 and 5.3 we can approximate the set $S_{A B}$ as an intersection of pseudohalfspaces in a suitable blow-up $\mathcal{B}$ of $S$. If we delete from $\mathcal{B}$ the original tropical pseudohyperplanes from $S$ we obtain a fine mixed subdivision. By the Topological Representation Theorem 4.12 the boundaries of these pseudohalfspaces yield an arrangement of affine pseudohyperplanes. Hence $S_{A B}$ is approximated by an intersection of closed affine pseudohalfspaces and hence is path-connected by Lemma 4.5

From this we immediately obtain the following corollary (cf. (Ardila and Develin 2009, Conj 5.1)):

Corollary 5.5 Tropical oriented matroids with parameters $(n, d)$ are in one-to-one correspondence with mixed subdivisions of $n \triangle^{d-1}$ and subdivisions of $\triangle^{n-1} \times \triangle^{d-1}$.

\section{References}

F. Ardila and M. Develin. Tropical hyperplane arrangements and oriented matroids. Mathematische Zeitschrift, 262(4):795-816, 2009.

A. Björner, M. Las Vergnas, B. Sturmfels, N. White, and G. M. Ziegler. Oriented matroids. Cambridge University Press, 1999.

J. A. De Loera, J. Rambau, and F. Santos. Triangulations: Applications, structures, algorithms. Springer, 2010.

M. Develin and B. Sturmfels. Tropical convexity. Doc. Math., 9:1-27, 2004.

M. Hochster. Rings of invariants of tori, cohen-macaulay rings generated by monomials, and polytopes. The Annals of Mathematics, 96(2):318-337, 1972.

S. Horn. Tropical Oriented Matroids and Cubical Complexes. PhD Thesis, TU Darmstadt, 2012.

B. Huber, J. Rambau, and F. Santos. The Cayley trick, lifting subdivisions and the Bohne-Dress theorem on zonotopal tilings. Journal of the European Mathematical Society, 2(2):179-198, 2000.

$\mathrm{S}$. Oh and H. Yoo. Triangulations of $\Delta_{n-1} \times \Delta_{d-1}$ and Tropical Oriented Matroids. arXiv:10094750v3 [math.CO], 2010.

E.-C. Zeeman. Seminar on combinatorial topology. Institut des Hautes Études Scientifiques, Fascicule 1 (Exposés I à V inclus), 1963. 